\title{
La lectura de textos no literarios en los textos escolares de lenguaje y comunicación de segundo ciclo básico del sistema escolar chileno: la apreciación de los profesores ${ }^{1}$
}

\author{
Beatriz Arancibia Gutiérrez
}

barancib@ucsc.cl

Marisol Henríquez Barahona ${ }^{2}$

mhenriquez@ucsc.cl

\section{RESUMEN}

En este artículo se dan a conocer los principales resultados de una investigación realizada en Chile durante 2006 y comienzos de 2007, cuyo propósito central fue averiguar cuál era la apreciación docente sobre la correspondencia de la propuesta de enseñanza de la lectura contenida en los libros de texto con el enfoque con que ésta es abordada por los programas de estudio. La investigación se focalizó en los textos escolares de segundo ciclo básico y los informantes fueron profesores del subsector Lenguaje y Comunicación de ese nivel escolar. Los resultados mostraron una tendencia a situar los textos en un nivel de mediana correspondencia con las bases conceptuales y programáticas de los programas.

\section{Palabras clave}

Comprensión de lectura, programas de estudio, segundo ciclo básico, textos escolares, textos no literarios, apreciación, profesores.

\section{Abstract}

This paper discusses the results of a research carried out in Chile during 2006 to early 2007 whose main purpose was to know the teachers' opinion about the correspondence between the textbooks proposal for the teaching of reading comprehension and the approach suggested by the National (Syllabus) Curriculum. The research focused on middle school textbooks, from $5^{\text {th }}$ to $8^{\text {th }}$ grade and teachers from the Language and Communication area. Results show a trend to regard textbooks as having a medium level of agreement with the conceptual approach present in the National (Syllabus) Curriculum.

\section{KEYWORDS}

Reading comprehension, middle school ( $5^{\text {th }}$ to $8^{\text {th }}$ grades), textbooks, non-literary texts, school teachers.

1 Universidad Católica de la Santísima Concepción, Chile. Investigación desarrollada a través del proyecto financiado por la Dirección de Investigación de la UCsC, DINCs\&H 03/2006.

2 Magísteres en Lingüística. Docentes del Departamento de Lengua y Literatura de la Facultad de Educación de la Universidad Católica de la Santísima Concepción. 


\section{Introducción}

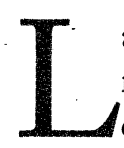

a lectura es una de las prácticàs escolares más habituales y el desarrollo de esta capacidad constituye una de las tareas centrales de la educación formal a nivel inicial. En Chile, el actual currículo para la enseñanza de lengua materna en el sistema escolar propone que al término de los ocho años de enseñanza básica los estudiantes sean capaces de leer con variados propósitos, de manera independiente, comprensiva y crítica, diversos tipos de textos. ${ }^{3}$ Para alcanzar este objetivo, los programas de estudio del subsector Lenguaje y Comunicación correspondientes al segundo ciclo básico (5० a $8^{\circ}$ año) incluyen la lectura de textos literarios y no literarios, ampliando paulatinamente el repertorio de estructuras textuales y de estrategias lectoras de los niños y las niñas.

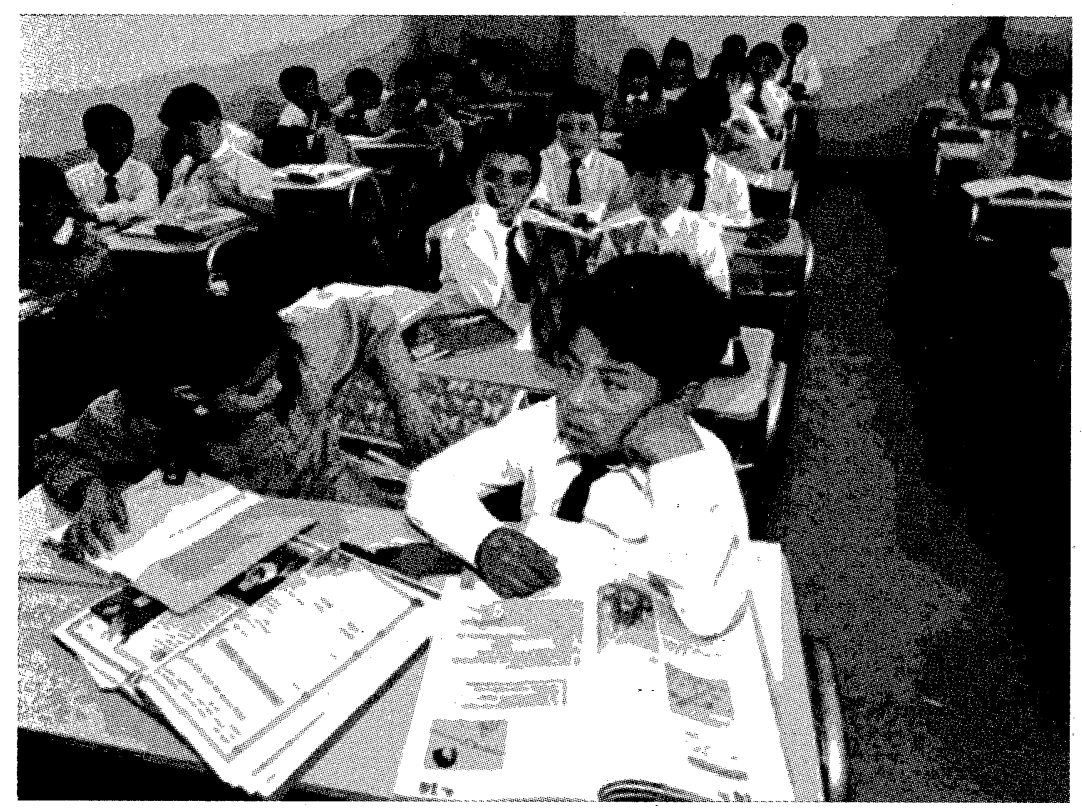

Como una manera de apoyar la implementación del nuevo currículo, instalado en el marco de la aplicación de la Reforma Educacional, el Ministerio de Educación impulsó durante el año

\footnotetext{
3 Lenguaje y Comunicación, Programa de Estudio $8^{\circ}$ año básico, NB6, p. 33 [en línea], disponible en: http:// www.curriculum-mineduc.cl/docs/fichas $/ 8$ b01 lenguaje_y_comunicacion.pdf. Véase también: http:// www.curriculum-mineduc.cl/docs/currEducBasi/ marco_curricular_de_educbasica.pdf, p. 69.
}

2000 el Programa Textos Escolares. Sus objetivos son contribuir a generar condiciones de mayor equidad en el acceso a materiales de estudio, apoyar el trabajo de los profesores y proporcionar una base común de conocimiento y herramientas para el aprendizaje. ${ }^{4}$ Según datos ministeriales, ${ }^{5}$ aproximadamente el $90 \%$ de los profesores de segundo ciclo básico utiliza estos textos, aunque se estima que sólo un $9 \%$ de los docentes los emplea como herramienta principal en la enseñanza. A partir de estos antecedentes nos surgió la inquietud como investigadoras por realizar un estudio que abordara la relación texto escolar-docente. Nos dimos entonces a la tarea de averiguar cuál era la opinión de los profesores de segundo ciclo sobre la correspondencia del tratamiento de la lectura en los textos escolares con el enfoque de la lectura presente en los programas de estudio. Nos apoyamos para ello en el trabajo realizado antes por Núñez y Donoso (2000), ${ }^{6}$ que consistió en el diseño y aplicación de una pauta de evaluación de la propuesta de enseñanza de la comprensión de las ideas principales contenida en los libros de Lengua Castellana y Comunicación de $1^{\circ}$ a $3^{\circ}$ medio.

Durante el segundo ciclo básico la lectura va adquiriendo un protagonismo creciente como medio a través del cual los alumnos y alumnas acceden a los contenidos de otros subsectores de

4 Información disponible en: http://portal.textosescolares. $\mathrm{cl} /$ website/index2.php?id_portal=1\&id_seccion=15\&id_ contenido $=48$.

5 Datos proporcionados por la Unidad Currículum y Evaluación del Ministerio de Educación en el "Seminario Disciplinar: Programa de Formación para la Apropiación Curricular" organizado por el Centro de Perfeccionamiento y Experimentación en Educación (CPEIP) y realizado el 29 de junio de 2005 . Actualmente la información está disponible en línea en: http://vilcun.datacare.cl/textosescolares/portal/documentos/admdocs/ docs/200801311147340. Resumen_Ejecutivo_Estudio_ Uso_de_Textos_2.pdf

6 Núñez, P. y Donoso, P. (2000), "Evaluación de la propuesta de enseñanza de la comprensión lectora en los textos escolares de la Reforma Educacional Chilena: un estudio exploratorio", en Signos, núm. 47, vol. 33, pp. 123-150. Estas autoras diseñaron una pauta de evaluación que entregaron a un grupo de profesores y alumnos de pedagogía con el objetivo de evaluar a través de sus opiniones la propuesta de enseñanza de la comprensión lectora contenida en los textos de Lengua Castellana y Comunicación de $1^{\circ}$ a $3^{\circ}$ de Enseñanza Media. 
aprendizaje. Los textos más usados con esta finalidad tienen características similares: predominio de la estructura expositiva, con secuencias descriptivas y argumentativas. Adèmás, en esta etapa escolar los niños y niñas interactúan cada vez más y con mayor autonomía con el entorno, lo que implica un mayor contacto con textos funcionales de uso cotidiano, incluidos los mensajes que circulan a través de los medios de comunicación. Por estas razones, decidimos focalizar nuestro trabajo en la lectura de textos no literarios.

\section{Marco de referencia}

\subsection{Concepto de lectura}

De acuerdo con lo declarado en el programa de $5^{\circ}$ básico, el concepto de lectura en que se basa el currículo de Lenguaje y Comunicación es coincidente con el enfoque interactivo de la lectura (1992, 2001). Ésta se concibe como "[...] un diálogo entre un lector y el texto escrito; es decir, un proceso de construcción de significados en el cual el lector interactúa con el texto estableciendo una relación entre sus conocimientos y experiencias previas con los aportes del texto" (programa Lenguaje y Comunicación, $5^{\circ}$ básico, p. 10).

El modelo que subyace a esta forma de entender la lectura es el desarrollado por van Dijk y Kintsch (1983), el cual, tal como expone $\mathrm{Pa}^{-}$ rodi $(2002$, p. 73$)$, se basa en dos supuestos fundamentales: el primero es de carácter cognitivo y enfatiza la importancia de los conocimientos previos del lector sobre el tema del texto y el proceso mismo de lectura. El uso adecuado de estos conocimientos durante la construcción del significado global del texto es la base de la interacción entre texto y lector. $\mathrm{El}$ segundo supuesto del modelo es que el procesamiento de un texto escrito, junto con ser una tarea cognitiva de gran complejidad, es también un evento psicosocial. El lector enfrenta la tarea de lectura como parte de una situación específica en un contexto sociocultural determinado. Por lo tanto, la compresión incluye la representación de los elementos contextuales y situacionales que permiten interpretar coherentemente las intenciones del discurso.

\subsection{El proceso de construcción de la representación del significado del texto: niveles de representación}

La suma de los significados de cada una de las oraciones de un texto no se corresponde con su significado global. Las operaciones que el sujeto realiza para construir el significado global se realizan en diferentes niveles y sus resultados son representaciones cada vez más elaboradas. Los niveles de representación mental del texto propuestos por van Dijk y Kintsch (1983), como es sabido, son los siguientes:

i) La representación lingüistica superficial, es decir, el recuerdo de las palabras y la estructura sintáctica de superficie de las oraciones. En general, éste resulta ser muy efímero y tiene poca importancia para la comprensión en niveles superiores, a menos que algún aspecto del código de superficie tenga mucha relevancia en el significado.

ii) La construcción de la representación semántica (de tipo proposicional) del texto: la microestructura y la macroestructura. La microestructura corresponde a las relaciones de sentido que se establecen a nivel local o entre oraciones adyacentes (texto base). La macroestructura, por su parte, consiste en la representación semántica global del texto mediante la construcción de macroproposiciones según un criterio jerárquico. Dicho en otras palabras, corresponde a la síntesis de las ideas más importantes y que le dan sentido al texto.

iii) El lector también construye una representación situacional o modelo mental de lo referido por el texto mediante un proceso inferencial basado en la interacción entre la información textual y su propio conocimiento del mundo: Además, durante la lectura el lector puede hacer uso de su conocimiento sobre los tipos de texto, lo que le permite articular la trama de interrelaciones causales, descriptivas, comparativas o temporales que mantienen las ideas globales en lo que conocemos como superestructura textual. 


\subsection{El papel del lector y el uso de estrategias de lectura}

El modelo de van Dijk y Kintsch (1983) no sólo se define por atribuirle al procesamiento del discurso escrito un carácter proposicional, sino además por asignarle un carácter estratégico. Ello se debe a que enfatiza el papel activo del sujeto, cuyas experiencias, conocimientos y propósitos de lectura le permiten interactuar con el texto y seleccionar de manera flexible, no prescriptiva, las estrategias para comprender mejor. De ahí que esta forma de entender el rol del lector ha tenido amplio eco en el ámbito de la enseñanza bajo el nombre de "enfoque interactivo" (1992, 2001). Desde esta perspectiva, el lector puede ir guiando y controlando sus propios procesos de lectura para llegar a construir el significado global del texto.

\subsection{La enseñanza de la lectura desde el enfoque interactivo}

La enseñanza de la lectura desde el enfoque interactivo implica el desarrollo de actitudes, conocimientos y capacidades que conduzcan a formar un lector autónomo, que es precisamente uno de los objetivos de la educación chilena en el segundo ciclo básico. ${ }^{7}$ Un lector autónomo es aquel que a través de su experiencia con la lectura desarrolla procesos metacomprensivos que lo llevan a tomar conciencia de que leer implica enfrentar un problema cuya resolución depende de que el plan de acción que emprenda sea adecuado a la tarea que debe llevar a cabo (Parodi y Núñez, 1998 y Solé, 2001). Según Peronard et al. (2002), esto significa que el lector debe contar con un conocimiento o conciencia acerca de la naturaleza de la tarea de lectura, vale decir, de su objetivo y las demandas cognitivas que conlleva. También debe tener conocimiento sobre las estrategias o acciones mentales que puede emprender antes, durante y después de leer para llevarla

\footnotetext{
Lenguaje y Comunicación, Programa de Estudio $8^{\circ}$ año básico, NB6, p. 33 [en línea], disponible en: http://www. curriculum-mineduc.cl/docs/fichas/8b01_lenguaje_y_ comunicacion.pdf. Véase también: http://www. curriculum-mineduc.cl/docs/currEducBasi/marco_curricular_de_educbasica.pdf.
}

a cabo y, por último, necesita una teoría o conocimiento sobre los textos y las tipologías textuales (véase también Crespo et al., 2003).

Además de las variables texto y lector, en el desarrollo de la capacidad para leer comprensivamente intervienen factores contextuales relacionados con la situación de lectura. En este sentido, resulta fundamental ofrecer a los alumnos y alumnas variadas experiencias de lectura con textos de diverso tipo y variadas funciones, más la enseñanza explícita de estrategias aplicables a dichas situaciones (véase Solé, 1992 y Condemarín, 2004).

La enseñanza de estrategias de lectura en el contexto de un modelo de enseñanza explícita requiere, entre otros, enfocar la comprensión como proceso y no sólo como producto medible a posteriori. Por esta razón suele ordenarse en tres fases: antes, durante y después de leer (Solé, 1992, 2001; Cassany et al., 2000, y Condemarín, 2004), como lo hacen los programas de Lenguaje y Comunicación. Antes de leer corresponde a una etapa de motivación hacia la lectura, fijación de objetivos, activación de los conocimientos previos de los alumnos (tema, tipo de texto) y formulación de predicciones y preguntas sobre el texto. Durante la lectura, las estrategias van encaminadas a comprobar las predicciones establecidas antes de leer, formular nuevas predicciones, evaluar la comprensión y corregir los posibles fallos. Después de la lectura, corresponde principalmente comprobar la comprensión de tema e ideas principales, y la inten-

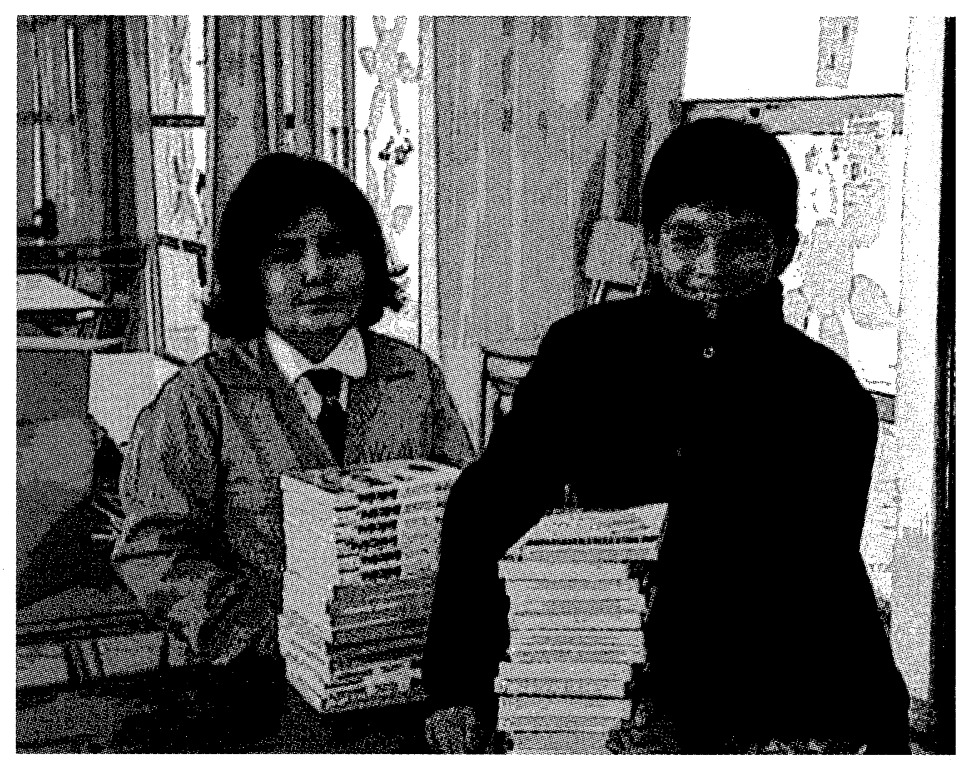


cionalidad del autor. Como se trata de un proceso dinámico, estas tareas pueden tener un carácter recursivo y no necesariamente seguir un trayecto lineal o esquemático, ya que la comprensión es un proceso continuo de construcción de significados desde el momento en que se inicia la lectura.

El repertorio de estrategias aplicables a cada fase del proceso lector es amplio, (véase Baumann, 1990; Condemarín, 2004; Sánchez, 1993, y Solé, 1992 y 2001). Aquí sólo mencionaremos aquellas cuya función es seleccionar y relacionar las ideas principales o macroproposiciones con las cuales el lector construye el sentido global del texto, las llamadas macrorreglas o macroestrategias de construcción semántica propuestas por van Dijk y Kintsch (1983): i) supresión de la información innecesaria o que resulta redundante; ii) selección de las proposiciones necesarias para comprender, que expresan un tópico de manera explícita en el texto; iii) generalización de dos o más proposiciones que puedèn ser reemplazadas por una de carácter más general, y iv) construcción de una nueva proposición a partir de información textual y de los conocimientos del lector por medio de la realización de inferencias.

Como señalan Núñez y Donoso (2000), comprender un texto consiste en captar la naturaleza jerárquica de su estructura semántica, por lo que manejar apropiadamente estas macroestrategias para poder llevarlo a cabo requiere de un proceso de lento aprendizaje que se caracteriza por su gran complejidad.

\section{Metodología}

Las preguntas que la investigación buscaba responder eran ¿cómo evalúan los docentes que usan los textos escolares la manera en que éstos abordan la lectura de textos no literarios?, ¿los textos escolares reflejan, a juicio de estos docentes, el enfoque teórico y la propuesta de enseñanza que plantean los programas de estudio respecto de la lectura?

\subsection{Participantes}

Los participantes fueron 39 docentes en ejercicio en el segundo ciclo básico en el subsector
Lenguaje y Comunicación en distintas escuelas de la provincia de Concepción. Su participación fue voluntaria y todos cumplían con el requisito de usar los textos escolares. El promedio de años de servicio profesional era de 11,8 años, de los cuales 6,5 los habían ejercido en Lenguaje y Comunicación. 25 de estos profesores estaban cursando o iniciando un postítulo de mención en el subsector. E1 69\% del total había antes realizado otros perfeccionamientos en el área.

\subsection{Descripción de los instrumentos y procedimientos empleados para recoger la información}

El primer paso en la investigación fue hacer un seguimiento de la lectura de textos no literarios a través de las distintas secciones de los programas de estudio (objetivos fundamentales, contenidos, aprendizajes esperados y orientaciones didácticas). La información seleccionada fue vaciada en tablas resumen, una para cada programa.

Posteriormente, se diseñó una breve encuesta cuyos propósitos fueron recoger información sobre los informantes y el uso que hacían de los textos. En concreto, se les preguntó con qué frecuencia y propósitos los empleaban, cuáles eran las secciones que más usaban y su evaluación de determinados aspectos.

Para responder las preguntas centrales de la investigación se usó una pauta construida como una escala de apreciación. Este instrumento fue elaborado con base en la pauta diseñada y aplicada por Núñez y Donoso (2000) para evaluar el tratamiento de la lectura en los textos escolares de Lengua Castellana y Comunicación de $1^{\circ}$ a $3^{\circ}$ medio. Dicha pauta consta de 41 preguntas agrupadas en 7 categorías: concepto y tipos de textos, conocimiento previo, objetivos de la lectura, jerarquía de las ideas, estrategias de lectura, metacomprensión y evaluación. Para efectos de la presente investigación, las preguntas se transformaron en enunciados afirmativos y se subagruparon en las siguientes 6 categorías, definidas de acuerdo con lo que señalan los programas de estudio y los lineamientos del enfoque interactivoestratégico de la lectura en el que se sustentan: 


\begin{tabular}{|c|c|}
\hline Categorías & Subcategorías \\
\hline \multirow{3}{*}{$\begin{array}{l}\text { Conceptos y tipos de } \\
\text { textos }\end{array}$} & 1. Variedad de lecturas de los tipos de textos que señala el programa de estudio. \\
\hline & 2. Explicaciones y actividades relacionadas con las estructuras de estos tipos de textos. \\
\hline & $\begin{array}{l}\text { 3. Coherencia conceptual en el tratamiento de los contenidos referidos a los textos y sus } \\
\text { características. }\end{array}$ \\
\hline \multirow{4}{*}{ Antes de la lectura } & $\begin{array}{l}\text { 1. Adecuación de los temas de las lecturas a los conocimientos e intereses de los alumnos } \\
\text { destinatarios. }\end{array}$ \\
\hline & 2. Activación del conocimiento previo de los alumnos relacionado con las lecturas. \\
\hline & 3. Actividades destinadas a formular predicciones sobre la lectura. \\
\hline & 4. Formulación de un objetivo asociado a las lecturas propuestas. \\
\hline \multirow{4}{*}{$\begin{array}{l}\text { Construcción de los } \\
\text { significados: estrategias } \\
\text { de procesamiento de la } \\
\text { información durante la } \\
\text { lectura }\end{array}$} & 1. Explicaciones y actividades basadas en estrategias para reconocer tema e ideas principales. \\
\hline & 2. Explicaciones y actividades basadas en estrategias para relacionar mejor la información. \\
\hline & $\begin{array}{l}\text { 3. Explicaciones y actividades basadas en estrategias para usar el conocimiento de las estructuras } \\
\text { textuales para comprender mejor la organización jerárquica de las ideas. }\end{array}$ \\
\hline & 4. Explicaciones y actividades destinadas a desarrollar capacidades para leer críticamente. \\
\hline \multirow{4}{*}{$\begin{array}{l}\text { Construcción de los } \\
\text { significados: estrategias } \\
\text { de procesamiento de la } \\
\text { información después de la } \\
\text { lectura }\end{array}$} & 1. Actividades dirigidas a dar cuenta de la comprensión del sentido global del texto. \\
\hline & $\begin{array}{l}\text { 2. Explicaciones y actividades relacionadas con estrategias de comprensión y recuerdo de la } \\
\text { información útiles al aprendizaje con la lectura. }\end{array}$ \\
\hline & $\begin{array}{l}\text { 3. Actividades destinadas a relacionar y evaluar lo leído con las propias experiencias y } \\
\text { conocimientos. }\end{array}$ \\
\hline & 4. Coherencia entre las actividades propuestas y el propósito de lectura. \\
\hline \multirow{5}{*}{$\begin{array}{l}\text { Metacomprensión: } \\
\text { reflexión sobre la lectura }\end{array}$} & $\begin{array}{l}\text { 1. Valoración de la lectura como medio para acceder al conocimiento, alcanzar autonomía y } \\
\text { eficacia en el aprendizaje. }\end{array}$ \\
\hline & $\begin{array}{l}\text { 2. Conciencia de que los conocimientos previos sobre el tema del texto son importantes para } \\
\text { comprender mejor las ideas. }\end{array}$ \\
\hline & $\begin{array}{l}\text { 3. Conciencia de que la lectura puede ser abordada de distintas maneras según los objetivos del } \\
\text { lector. }\end{array}$ \\
\hline & $\begin{array}{l}\text { 4. Conciencia de la aplicación de determinadas estrategias para comprender mejor el significado } \\
\text { del texto. }\end{array}$ \\
\hline & 5. Actividades dirigidas a autoevaluar el proceso de identificación de las ideas. \\
\hline \multirow{2}{*}{$\begin{array}{l}\text { Evaluación de la } \\
\text { comprensión }\end{array}$} & $\begin{array}{l}\text { 1. Evaluación de la comprensión en diferentes niveles de generalización de los contenidos de los } \\
\text { textos. }\end{array}$ \\
\hline & 2. Retroalimentación de las respuestas del lector. \\
\hline
\end{tabular}

Tabla 1: Categorías y subcategorías para el diseño de la pauta.

El instrumento quedó conformado finalmente por 34 afirmaciones, algunas de las cuales se redactaron con una leve diferencia para cada nivel escolar con el fin de hacer énfasis en las particularidades de los programas. Por ejemplo, la primera afirmación del bloque "Conceptos y tipos de textos" especifica según el curso los tipos de textos que señalan los programas. Así, en la pauta usada para $7^{\circ}$ básico este enunciado dice "E1 libro escolar contiene variadas lecturas de los tipos de textos que señala el programa de estudio de 70 año: informativos, normativos, publicitarios, instrumentales y argumentativos".

La tarea solicitada a los profesores informantes fue manifestar su nivel de acuerdo con lo afirmado asignando un puntaje dẹ 1 a 5 como lo indica el siguiente cuadro:

\begin{tabular}{|c|l|}
\hline Puntaje & \multicolumn{1}{|c|}{ Nivel de acuerdo } \\
\hline 1 & Muy en desacuerdo \\
\hline 2 & En desacuerdo \\
\hline 3 & Medianamente de acuerdo \\
\hline 4 & De acuerdo \\
\hline 5 & Totalmente de acuerdo \\
\hline
\end{tabular}

Para el análisis de la información se empleó un procedimiento similar a una escala de apreciación Likhert. El puntaje otorgado a cada enunciado fue acumulativo y se promedió por el 
número de respuestas (39). Los datos cuantitativos se tradujeron en niveles de correspondencia del texto escolar con los lineamientos teóricos y programáticos de los programas dè estudio, como lo muestra el cuadro a continuación:

\begin{tabular}{|c|l|}
\hline Promedio & \multicolumn{1}{|c|}{ APRECIACIÓN } \\
\hline $1-2$ & No hay correspondencia \\
\hline $2,1-3$ & Hay baja correspondencia \\
\hline $3,1-4$ & Hay mediana correspondencia \\
\hline $4,1-5$ & Hay alta correspondencia \\
\hline
\end{tabular}

Cada profesor recibió una copia de la tabla resumen de los programas de $5^{\circ}$ a $8^{\circ}$ como una manera de asegurarnos de que respondieran teniendo en cuenta lo que éstos señalan. Respondieron en forma individual en un plazo mutuamente acordado con las investigadoras.

\section{Resultados}

\subsection{Uso de los textos escolares}

En su conjunto, 28 de los participantes señalaron que usaban el texto escolar "siempre" o "casi siempre" (71.7\%). Con respecto al propó- sito, "trabajo en aula" acumuló el mismo número de respuestas (28). Sólo 7 docentes del total (17.9\%) señalaron que usaban el texto cuando preparaban sus clases. Sin duda, esto resulta inquietante y nos plateó nuevas interrogantes, toda vez que al ser consultados sobre qué era lo que preferían usar de los textos la respuesta mayoritaria fue "las lecturas", alternativa que ocupó el primer lugar de preferencias. En cambio, "sugerencias de evaluación" ocupó el último lugar. Estas respuestas dieron origen a un objetivo emergente que fue averiguar a través de entrevistas en profundidad a un subgrupo de profesores informantes cómo trabajaban y evaluaban la lectura en aula y sobre qué bases conceptuales lo hacían. En esta oportunidad, nos limitamos a informar los resultados de la investigación principal.

\subsection{Apreciación de la correspondencia entre los textos escolares y los programas de estudio}

En la siguiente tabla resumen se presentan los promedios obtenidos por cada categoría y su traducción conceptual de acuerdo a la escala de apreciación empleada. Por razones de espacio no se presenta un análisis pormenorizado por enunciado.

\begin{tabular}{|c|c|c|c|c|c|c|c|c|c|c|}
\hline \multirow{2}{*}{ Categoría } & \multicolumn{2}{|c|}{$5^{\circ}$} & \multicolumn{2}{|c|}{$6^{\circ}$} & \multicolumn{2}{|c|}{$7^{\circ}$} & \multicolumn{2}{|c|}{$8^{\circ}$} & \multicolumn{2}{|c|}{ Global/aspecto } \\
\hline & Prom. & Corresp. & Prom. & Corresp. & Prom. & Corresp. & Prom. & Corresp. & Prom. & Corresp \\
\hline Conceptos y tipos de textos & 3,42 & Media & 3,96 & Media & 3,22 & Media & 3,44 & Media & 3,51 & Media \\
\hline Antes de la lectura & 3,1 & Media & 3,67 & Media & $-3,12$ & Media & 2,97 & Baja & 3,21 & Media \\
\hline $\begin{array}{l}\text { Construcción de los } \\
\text { significados: estrategias } \\
\text { de procesamiento de la } \\
\text { información durante la lectura }\end{array}$ & 2,92 & Baja & 3,34 & Media & 3 & Baja & 2,92 & Baja & 3,05 & Baja \\
\hline $\begin{array}{l}\text { Construcción de los } \\
\text { significados: estrategias } \\
\text { de procesamiento de la } \\
\text { información después de la } \\
\text { lectura }\end{array}$ & 2,94 & Baja & 3,31 & Media & 3,17 & Media & 3,02 & Baja & 3,11 & Media \\
\hline $\begin{array}{l}\text { Metacomprensión: reflexión } \\
\text { sobre la lectura }\end{array}$ & 2,77 & Baja & 4,21 & Alta & 2,99 & Baja & 2,59 & Baja & 3,14 & Media \\
\hline Evaluación de la comprensión & 2,78 & Baja & 3,26 & Media & 2,74 & Baja & 2,79 & Baja & 2,89 & Baja \\
\hline Global/curso & 2,98 & Baja & 3,63 & Media & $3 ; 04$ & Baja & 2,95 & Baja & 3,15 & Media \\
\hline
\end{tabular}

Tabla 2: Apreciación de la correspondencia entre los textos escolares y los programas de estudio én relación con la lectura de textos no literarios (por nivel escolar y dimensión). 
Un examen global de los datos por curso muestra que, aunque en general los promedios estuvieron muy próximos a alcanzar el valor mínimo de 3,1 para estar en el rango "mediana correspondencia”, el único texto que logró ese nivel de valoración fue el de $6^{\circ}$ básico, con un promedio de 3,63. De hecho, sólo este mismo texto alcanzó en una de las categorías (metacomprensión) el rango "alta correspondencia" con los lineamientos del Programa. Por su parte, el texto escolar que obtuvo una evaluación más baja fue el de $8^{\circ}$, que sólo en "Conceptos y tipos de texto" se ubicó en el rango de "mediana correspondencia".

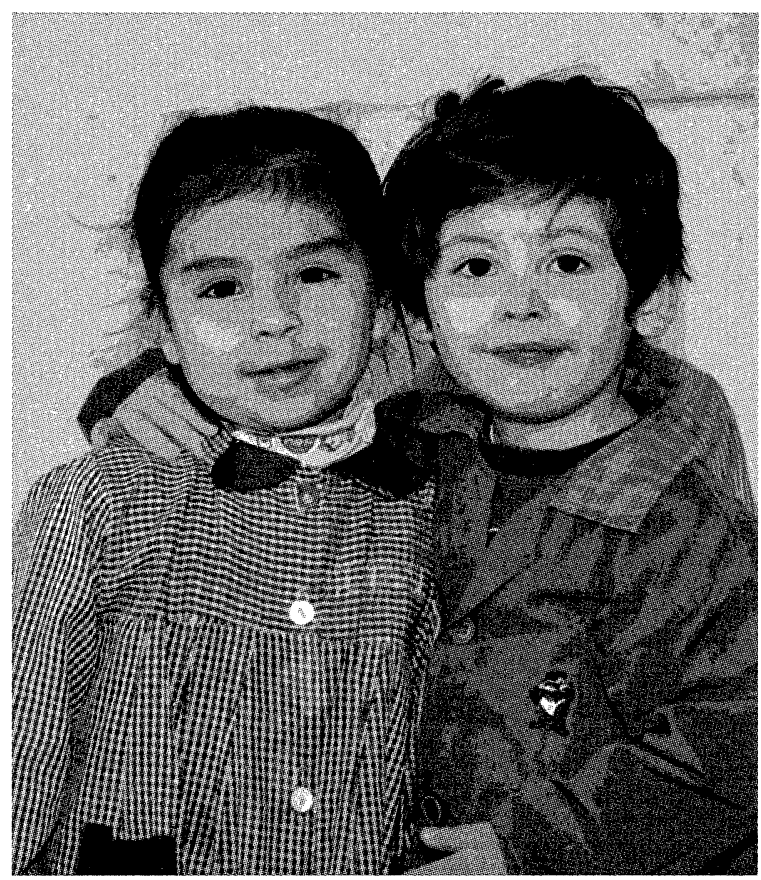

Al mirar los datos por categoría, se observa una tendencia general hacia un nivel de "mediana correspondencia”, aunque en su mayoría los promedios están muy próximos al valor límite de 3,1. Ahora bien, la categoría mejor evaluada en términos globales y por curso fue "Conceptos y tipos de texto" $(3,51)$. Este resultado coincide con el obtenido por Núñez y Donoso (2000) en la evaluación realizada a los libros de $1^{\circ}$ a $3^{\circ}$ de enseñanza media. Al igual que estas autoras, pensamos que esto refleja la importancia que se le otorga actualmente a los conocimientos del lector sobre las estructuras textuales. Esto es notable en los programas de estudio, que presentan los contenidos referidos a la lectura organizados por tipos de texto. Sin embargo, un examen más detallado nos advirtió que, según la opinión de los profesores evaluadores, dicha preocupación se inscribe principalmente en el campo de los contenidos conceptuales, pero no se refleja a nivel procedimental de la misma manera. Este juicio se basa en el bajo promedio obtenido por uno de los enunciados referidos al uso de estrategias: "El texto escolar enseña estrategias para usar el conocimiento de las estructuras textuales para comprender mejor", que alcanzó un nivel de acuerdo de 2,85 puntos en promedio.

Un resultado que nos provocó preocupación fue el de la categoría "Construcción de los significados: estrategias de procesamiento de la información durante la lectura", que sólo en el libro de $6^{\circ}$ año traspasó los 3,1 puntos. En esta categoría quedaron agrupados los enunciados de la pauta referidos principalmente a estrategias destinadas a identificar el tema y discriminar las ideas más importantes durante la lectura. Al parecer, según la opinión de los profesores, los textos escolares dan por sabido que los niños y niñas saben reconocer lo principal de lo accesorio mientras leen o bien que los profesores están capacitados para enseñarles cómo hacerlo.

\section{Un lector autónomo es aquel que a través de su experiencia con la lectura desarrolla procesos metacomprensivos que lo llevan a tomar conciencia de que leer implica enfrentar un problema cuya resolución depende de que el plan de acción que emprenda sea adecuado a la tarea que debe llevar a cabo (Parodi y Núnez, 1998 y Solé, 2001).}

El nivel de acuerdo más bajo de los docentes fue con los enunciados de la categoría "Evaluación de la comprensión", que -en su conjuntopromediaron 2,81 puntos. Coincidentemente, en Núñez y Donoso (2000) este aspecto fue uno de los que obtuvo una evaluación más baja, 3,47 en una escala de 1 a 7 . 
Una observación importante es que si bien es cierto que la categoría "Metacomprensión" obtuvo un promedio global de 3,14 , es decir, "mediana correspondencia", ello se debe a ùn efecto de "arrastre" provocado por la puntuación alcanzada por el texto de $6^{\circ}$ básico $(4,21)$, ya que todos los otros estuvieron bajo los 3,1 puntos, particularmente el de $8^{\circ}(2,59)$. Visto así, la tendencia es también similar a la encontrada en el trabajo de Núñez y Donoso ya citado, en el que la misma categoría obtuvo una evaluación igual a 2,58 , la más baja de todos los aspectos evaluados.

En síntesis, podemos concluir que:

1. La apreciación de los profesores respecto de la correspondencia de los libros escolares de Lenguaje y Comunicación de segundo ciclo básico con la propuesta de enseñanza de la lectura de textos literarios contenida en los programas de estudio, en términos globales, es de "mediana correspondencia".

2. Visto en el contínuum de la pauta de apreciación empleada, este nivel de "mediana correspondencia" se encuentra en una zona limítrofe con el nivel de "baja correspondencia”. Usar el mismo instrumento con una muestra mayor de profesores evaluadores podría inclinar la balanza más claramente en uno u otro sentido.

3. El texto escolar mejor evaluado fue el de $6^{\circ}$ básico, el único que se ubicó en el rango "mediana correspondencia"; el que obtuvo la evaluación más baja fue el de $8^{\circ}$.

4. Ningún texto se ubicó en el rango "alta correspondencia".

5. La categoría que arrojó una mejor correspondencia con los lineamientos del currículo referidos a la lectura de textos no literarios fue "Conceptos y tipos de textos", en cambio "Evaluación de la comprensión" obtuvo el promedio más bajo.

6. En términos globales, ninguna categoría logró el puntaje requerido para alcanzar el nivel de "alta correspondencia". De hecho, ese resultado sólo se dio para el libro de $6^{\circ}$ básico en una categoría.

7. Los datos recogidos pueden ayudar a explicar por qué los profesores mayoritariamente respondieron que no preparaban sus clases con los textos escolares. Probablemente una valoración más alta del conjunto de las categorías se reflejaría en un mayor uso de estos textos en la programación de la enseñanza. Del mismo modo, la baja puntuación otorgada a la categoría "evaluación" permite entender mejor por qué estos profesores al ser consultados por las secciones del texto escolar que más empleaban con sus estudiantes dejaron las sugerencias de evaluación contenidas en los libros en el último lugar de sus preferencias.

8. Finalmente, las coincidencias con los resultados de la evaluación de los textos de Lengua Castellana y Comunicación encontrados en la investigación de Núñez y Donoso (2000) invitan a profundizar en el estudio y revisión del tratamiento de la lectura en los textos escolares chilenos tanto en el nivel básico como de enseñanza media. Ó

\section{Bibliografía}

Baumann, J. F. (1990), "La enseñanza directa de la habilidad de comprensión de la idea principal”, en Baumann, J.F. (edit.), La comprensión lectora (cómo trabajar la idea principal en el aula), Madrid, Aprendizaje/Visor, pp.133-173.

Cassany, D., Luna, M. y Sanz, G. (2000), Enseñar lengua, Barcelona, Editorial Graó.

Condemarín, M. y Medina, A. (2004), Taller de lenguaje II. Un programa integrado de desarrollo de las competencias lingüisticas y comunicativas de los alumnos de segundo ciclo básico, Santiago, Santillana.

Crespo, N.; García, G. y Carvajal. C. (2003), "Concepciones didácticas de la lectura, su influencia en el saber de los escolares", en Onomazein, vol. 8, PUc.

Ministerio de Educación, República de Chile. Educación Básica, Unidad de Currículum y Evaluación (2001). Lenguaje y Comunicación, Programa de Estudio $8^{\circ}$ año básico, NB6 [en línea], disponible en: http://www. curriculum-mineduc.cl/curriculum/programas-de-estudios/educacion-basica/

- (2001). Lenguaje y Comunicación, Programa de Estudio $5^{\circ}$ año básico, NB6 [en línea], 
disponible en: http://www.curriculum-mineduc.cl/curriculum/programas-de-estudios/educacion-basica/

Núñez, P. y Donoso, P. (2000), "Evaluàción de la propuesta de enseñanza de la comprensión lectora en los textos escolares de la Reforma Educacional Chilena: un estudio exploratorio", en Signos, núm. 47, vol. 33, pp. 123-150.

Parodi, G. (2002), "Comprensión lingüística: Hacia dónde vamos desde donde estamos?", en Parodi, G. (edit.), Lingüistica e interdisciplinariedad: Desafios del nuevo milenio. Ensayos en honor a Marianne Peronard, Valparaíso, Editorial Universitaria de la Ucv, pp. 44-67.

- y Núñez, P. (1998), "El desarrollo de estrategias de lectura comprensiva: Una aplicación experimental del Programa L y C: Leer y Comprender", en Peronard, M. et al., Comprensión de textos escritos: de la teoría a la sala de clases, Santiago, Editorial Andrés Bello, pp. 249-264.

Peronard, M., et al. (2002), "Conocimiento metacognitivo del lenguaje escrito: instrumento de medida y fundamentación Teórica", en Infancia y Aprendizaje, vol. 25, núm. 2, pp. 131-143.

Sánchez, E. (1993), Los textos expositivos. Estrategias para mejorar su comprensión, Madrid, Santillana.

Solé, I. (1992), Estrategias de lectura, Barcelona, Graó.

- (2001), “De la lectura al aprendizaje”, en Lomas, A. C. (comp.), El aprendizaje de la comunicación en las aulas, Barcelona, Paidós, pp. 107-122.

van Dijk, T. y Kintsch, W. (1983), Strategies of Discourse Comprehension, Nueva York, Academic Press. 